\title{
Taxonomic voucher specimens for study of post-wildfire forest habitat in Douglas County, Oregon
}

\author{
Sara M. Galbraith ${ }^{1}$, James H. Cane ${ }^{2}$, Andrew R. Moldenke ${ }^{3}$ Lincoln R. Best $^{4}$ and James W. Rivers ${ }^{1}$ \\ ${ }^{1}$ Department of Forest Ecosystems and Society, Oregon State University \\ ${ }^{2}$ USDA-ARS Pollinating Insects Research Unit, Logan, UT \\ ${ }^{3}$ Department of Botany and Plant Pathology, Oregon State University \\ ${ }^{4}$ Oregon State Athropod Collection, Oregon State University; rbest@gmail.com
}

Cite this work as:

Galbraith, S. M., J. H. Cane, A. R. Moldenke, L. R. Best and J. W. Rivers. 2019. Taxonomic voucher specimens for study of postwildfire forest habitat in Douglas County, Oregon. Catalog: Oregon State Arthropod Collection. 3(4) p. 1-12.

DOI: http://dx.doi.org/10.5399/osu/cat osac.3.4.4618.

\begin{abstract}
Post-disturbance salvage logging has negative consequences on certain components of forest biodiversity, but few studies have looked at how early seral-adapted organisms are influenced in salvage-logged areas while controlling for local severity of the wildfire. In this study (Galbraith et al., in revision), we investigated the influence of an essential group of pollinators - wild bees - to recent post-wildfire salvage logging within managed mixed-conifer forest in the Pacific Northwestern U.S. We compared bee communities and relevant habitat features in salvage-logged areas to unlogged sites, both of which experienced high-severity fire. These specimens- collected using Blue Vane Traps- were identified to the lowest possible taxonomic level by A. R. Moldenke and L. R. Best. Three hundred and ninety two taxonomic voucher specimens were deposited into the Oregon State Arthropod Collection to serve as a reference for future research (Accession: OSAC_AC_2019-09-10-01001).
\end{abstract}

\section{Description of the collection}

We collected bees and sampled bee habitat characteristics in 17 severely burned forest sites within the Douglas Fire Complex (Table 1) as part of a larger study investigating the influence of wildfire severity on wild bees (Galbraith et. al., 2019). Sampling took place during four sampling rounds each in May through September 2016 and 2017. During each collection period, we sampled bee communities using blue vane traps (BVTs; Springstar Inc., Woodinville, WA). At each sampling site, we hung two traps with no killing agent or preservative, each on $1.8 \mathrm{~m}$ tall T-posts. We left traps for $48 \mathrm{~h}$, after which we removed them and placed them into a cooler with dry ice to kill captured insects, then froze samples until processing in the lab.

We assigned each captured bee to genus using keys from Michener et al. (2007) and Stephen et al. (1969). Species-level identifications were determined where keys were available for the following genera: Agapostemon (Stephen et al., 1969), Anthophora and Ceratina (www.discoverlife.org), Bombus (Williams et al., 2014), Halictus (Roberts, 1973), and Xylocopa (Hurd and Moure, 1963). Remaining species-level identifications were made by Andrew Moldenke and Lincoln Best. No species keys were available for several genera in our region (e.g., Lasioglossum (Dialictus) and some Osmia); specimens in these genera were grouped to morphospecies. 
Table 1. List of sampling locations within the Douglas Fire Complex (UTM 10N)

\begin{tabular}{|c|c|l|}
\hline Site ID number & Post-wildfire treatment & UTM coordinates \\
\hline 4 & Unlogged & 10 N 4529514728980 \\
\hline 6 & Unlogged & 10 N 4557124729922 \\
\hline 7 & Unlogged & 10 N 4598644728947 \\
\hline 8 & Unlogged & 10 N 4578754730556 \\
\hline 27 & Unlogged & 10 N 4541134728147 \\
\hline 28 & Unlogged & 10 N 4517514737841 \\
\hline 29 & Unlogged & 10 N 4509364733986 \\
\hline 39 & Unlogged & 10 N 455995 4726212 \\
\hline 42 & Unlogged & 10 N 4495064735955 \\
\hline 1 & Logged & 10 N 4554344728316 \\
\hline 3 & Logged & 10 N 4575194731092 \\
\hline 9 & Logged & 10 N 4561634729656 \\
\hline 11 & Logged & 10 N 4584174734234 \\
\hline 26 & Logged & 10 N 4552654727319 \\
\hline 31 & Logged & 10 N 4603024727944 \\
\hline 38 & Logged & 10 N 4567934726957 \\
\hline 41 & Logged & 10 N 4479944735111 \\
\hline
\end{tabular}

We collected a total 3,561 specimens representing 21 genera and 111 morphospecies of bees. Of these, 65 were identified as known species and 46 were grouped by morphospecies (mostly within subgenus Lasioglossum (Dialictus)). Bees of different sexes could often not be matched at the morphospecies level, which inflated the number of morphospecies in our collection. See Galbraith et al. (in revision) for a list of abundances of each species.

Up to five specimens per sex per species were selected for the voucher collection. Individual-level data including the specimen's unique identification number, assigned species or morphospecies, and sex are provided in Table 2 . 


\begin{tabular}{|c|c|c|c|c|}
\hline identification & $\begin{array}{l}\text { date } \\
\text { collected }\end{array}$ & sex & labID & $\begin{array}{l}\text { Oregon State Arthropod Collection } \\
\text { UniquID }\end{array}$ \\
\hline Andrena prunorum Cockerell, 1896 & 08.vii.2016 & $\mathrm{F}$ & 369 & http:/ / osac.oregonstate.edu/SP/OSAC_0001226781 \\
\hline Andrena morphospecies \#1 & 30.vi.2016 & $\mathrm{F}$ & 534 & http:/ / osac.oregonstate.edu/SP/OSAC_0001226782 \\
\hline Andrena morphospecies \#1 & 04.viii.2016 & $\mathrm{F}$ & 758 & http:/ / osac.oregonstate.edu/SP/OSAC_0001226783 \\
\hline Andrena morphospecies \#2 & 26.v.2016 & $\mathrm{F}$ & 92 & http:/ / osac.oregonstate.edu/SP/OSAC_0001226784 \\
\hline Andrena morphospecies \#2 & 22.vi.2017 & $\mathrm{F}$ & 4276 & http:/ / osac.oregonstate.edu/SP/OSAC_0001226785 \\
\hline Andrena morphospecies \#2 & 29.vi.2017 & $\mathrm{F}$ & 4651 & http:/ / osac.oregonstate.edu/SP/OSAC_0001226786 \\
\hline Andrena morphospecies \#4 & 28.vi.2017 & $\mathrm{F}$ & 4502 & http:/ / osac.oregonstate.edu/SP/OSAC_0001226787 \\
\hline Andrena morphospecies \#5 & 08.vii.2016 & $\mathrm{F}$ & 371 & http:/ / osac.oregonstate.edu/SP/OSAC_0001226788 \\
\hline Andrena morphospecies \#5 & 30.vi.2016 & $\mathrm{F}$ & 721 & http:/ / osac.oregonstate.edu/SP/OSAC_0001226789 \\
\hline Andrena morphospecies \#7 & 25.v.2016 & $\mathrm{F}$ & 162 & http:/ / osac.oregonstate.edu/SP/OSAC_0001226790 \\
\hline Andrena morphospecies \#9 & 18.v.2017 & $\mathrm{F}$ & 3752 & http:/ / osac.oregonstate.edu/SP/OSAC_0001226791 \\
\hline Perdita nevadensis Cockerell, 1896 & 03.viii.2016 & $\mathrm{F}$ & 1863 & http:/ / osac.oregonstate.edu/SP/OSAC_0001227277 \\
\hline Perdita nevadensis Cockerell, 1896 & 03.viii.2016 & $\mathrm{F}$ & 2414 & http:/ / osac.oregonstate.edu/SP/OSAC_0001227278 \\
\hline Perdita nevadensis Cockerell, 1896 & 03.viii.2016 & $\mathrm{F}$ & 2436 & http:/ / osac.oregonstate.edu/SP/OSAC_0001227279 \\
\hline Perdita nevadensis Cockerell, 1896 & 28.vi.2017 & $\mathrm{F}$ & 4697 & http:/ / osac.oregonstate.edu/SP/OSAC_0001227280 \\
\hline \multicolumn{5}{|c|}{ APIDAE } \\
\hline Anthophora bomboides Kirby, 1837 & 27.vii.2017 & $\mathrm{F}$ & 5391 & http:/ / osac.oregonstate.edu/SP/OSAC_0001226799 \\
\hline Anthophora pacifica Cresson, 1878 & 19.v.2016 & $\mathrm{F}$ & 114 & http:/ / osac.oregonstate.edu/SP/OSAC_0001226803 \\
\hline Anthophora pacifica Cresson, 1878 & 19.v.2016 & $\mathrm{F}$ & 118 & http:/ / osac.oregonstate.edu/SP/OSAC_0001226804 \\
\hline Anthophora pacifica Cresson, 1878 & 25.v.2017 & $\mathrm{F}$ & 3703 & http:/ / osac.oregonstate.edu/SP/OSAC_0001226800 \\
\hline Anthophora pacifica Cresson, 1878 & 24.v.2017 & $\mathrm{F}$ & 3834 & http:/ / osac.oregonstate.edu/SP/OSAC_0001226801 \\
\hline Anthophora pacifica Cresson, 1878 & 23.vi.2017 & $\mathrm{F}$ & 4103 & http:/ / osac.oregonstate.edu/SP/OSAC_0001226802 \\
\hline Anthophora urbana Cresson, 1878 & 30.vi.2016 & $\mathrm{F}$ & 494 & http:/ / osac.oregonstate.edu/SP/OSAC_0001226807 \\
\hline Anthophora urbana Cresson, 1878 & 07.vii.2016 & $\mathrm{F}$ & 630 & http:/ / osac.oregonstate.edu/SP/OSAC_0001226809 \\
\hline Anthophora urbana Cresson, 1878 & 11.viii.2016 & $\mathrm{F}$ & 2741 & http:/ / osac.oregonstate.edu/SP/OSAC_0001226805 \\
\hline Anthophora urbana Cresson, 1878 & 11.viii.2016 & $\mathrm{F}$ & 2907 & http:/ / osac.oregonstate.edu/SP/OSAC_0001226806 \\
\hline Anthophora urbana Cresson, 1878 & 01.ix.2017 & $\mathrm{F}$ & 6131 & http:/ / osac.oregonstate.edu/SP/OSAC_0001226808 \\
\hline Apis mellifera Linnaeus, 1758 & 04.viii.2016 & $\mathrm{F}$ & 1136 & http:/ / osac.oregonstate.edu/SP/OSAC_0001226810 \\
\hline Apis mellifera Linnaeus, 1758 & 04.viii.2016 & $\mathrm{F}$ & 1142 & http:/ / osac.oregonstate.edu/SP/OSAC_0001226811 \\
\hline Apis mellifera Linnaeus, 1758 & 04.viii.2016 & $\mathrm{F}$ & 1145 & http:/ / osac.oregonstate.edu/SP/OSAC_0001226812 \\
\hline Apis mellifera Linnaeus, 1758 & 04.viii.2016 & $\mathrm{F}$ & 1146 & http:/ / osac.oregonstate.edu/SP/OSAC_0001226813 \\
\hline Apis mellifera Linnaeus, 1758 & 04.viii.2016 & $\mathrm{F}$ & 1147 & http:/ / osac.oregonstate.edu/SP/OSAC_0001226814 \\
\hline Bombus caliginosus (Frison, 1927) & 27.v.2016 & $\mathrm{F}$ & 331 & http:/ / osac.oregonstate.edu/SP/OSAC_0001226821 \\
\hline Bombus caliginosus (Frison, 1927) & 08.vii.2016 & $\mathrm{M}$ & 352 & http:/ / osac.oregonstate.edu/SP/OSAC_0001226825 \\
\hline Bombus caliginosus (Frison, 1927) & 10.viii.2016 & $\mathrm{F}$ & 2843 & http:/ / osac.oregonstate.edu/SP/OSAC_0001226817 \\
\hline Bombus caliginosus (Frison, 1927) & 11.viii.2016 & $\mathrm{F}$ & 2874 & http:/ / osac.oregonstate.edu/SP/OSAC_0001226818 \\
\hline Bombus caliginosus (Frison, 1927) & 11.viii.2016 & $\mathrm{F}$ & 2900 & http:/ / osac.oregonstate.edu/SP/OSAC_0001226819 \\
\hline Bombus caliginosus (Frison, 1927) & 11.viii.2016 & $\mathrm{M}$ & 2902 & http:/ / osac.oregonstate.edu/SP/OSAC_0001226822 \\
\hline Bombus caliginosus (Frison, 1927) & 10.viii.2016 & $\mathrm{M}$ & 2976 & http:/ / osac.oregonstate.edu/SP/OSAC_0001226823 \\
\hline
\end{tabular}




\begin{tabular}{|c|c|c|c|c|}
\hline Bombus caliginosus (Frison, 1927) & 10.viii.2016 & $\mathrm{M}$ & 2977 & http:/ / osac.oregonstate.edu/SP/OSAC_0001226824 \\
\hline Bombus caliginosus (Frison, 1927) & 10.viii.2016 & $\mathrm{F}$ & 2978 & http:/ / osac.oregonstate.edu/SP/OSAC_0001226820 \\
\hline Bombus caliginosus (Frison, 1927) & 22.vi.2017 & M & 4303 & http:/ / osac.oregonstate.edu/SP/OSAC_0001226826 \\
\hline Bombus fervidus (Fabricius, 1798) & 11.viii.2016 & M & 2877 & http:/ / osac.oregonstate.edu/SP/OSAC_0001226827 \\
\hline Bombus fervidus (Fabricius, 1798) & 07.vii.2016 & $\mathrm{F}$ & 446 & http:/ / osac.oregonstate.edu/SP/OSAC_0001226828 \\
\hline Bombus fervidus (Fabricius, 1798) & 23.vi.2017 & $\mathrm{M}$ & 4074 & http:/ / osac.oregonstate.edu/SP/OSAC_0001226829 \\
\hline Bombus fervidus (Fabricius, 1798) & 22.vi.2017 & $\mathrm{M}$ & 4249 & http:/ / osac.oregonstate.edu/SP/OSAC_0001226830 \\
\hline Bombus fervidus (Fabricius, 1798) & 22.vi.2017 & $\mathrm{M}$ & 4251 & http:/ / osac.oregonstate.edu/SP/OSAC_0001226831 \\
\hline Bombus fervidus (Fabricius, 1798) & 22.vi.2017 & M & 4252 & http:/ / osac.oregonstate.edu/SP/OSAC_0001226832 \\
\hline Bombus flavifrons Cresson, 1863 & 30.vi.2016 & $\mathrm{F}$ & 561 & http:/ / osac.oregonstate.edu/SP/OSAC_0001226837 \\
\hline Bombus flavifrons Cresson, 1863 & 07.vii.2016 & $\mathrm{M}$ & 627 & http:/ / osac.oregonstate.edu/SP/OSAC_0001226838 \\
\hline Bombus flavifrons Cresson, 1863 & 11.viii.2016 & $\mathrm{F}$ & 2873 & http:/ / osac.oregonstate.edu/SP/OSAC_0001226834 \\
\hline Bombus flavifrons Cresson, 1863 & 11.viii.2016 & $\mathrm{F}$ & 2881 & http:/ / osac.oregonstate.edu/SP/OSAC_0001226833 \\
\hline Bombus flavifrons Cresson, 1863 & 29.vi.2017 & $\mathrm{F}$ & 4462 & http:/ / osac.oregonstate.edu/SP/OSAC_0001226835 \\
\hline Bombus flavifrons Cresson, 1863 & 29.vi.2017 & $\mathrm{F}$ & 4567 & http:/ / osac.oregonstate.edu/SP/OSAC_0001226836 \\
\hline Bombus melanopygus Nylander, 1848 & 26.v.2016 & $\mathrm{F}$ & 319 & http:/ / osac.oregonstate.edu/SP/OSAC_0001226839 \\
\hline Bombus melanopygus Nylander, 1848 & 27.v.2016 & $\mathrm{M}$ & 334 & http:/ / osac.oregonstate.edu/SP/OSAC_0001226844 \\
\hline Bombus melanopygus Nylander, 1848 & 27.v.2016 & $\mathrm{M}$ & 335 & http:/ / osac.oregonstate.edu/SP/OSAC_0001226845 \\
\hline Bombus melanopygus Nylander, 1848 & 18.v.2017 & $\mathrm{F}$ & 3795 & http:/ / osac.oregonstate.edu/SP/OSAC_0001226840 \\
\hline Bombus melanopygus Nylander, 1848 & 25.v.2017 & $\mathrm{F}$ & 3896 & http:/ / osac.oregonstate.edu/SP/OSAC_0001226841 \\
\hline Bombus melanopygus Nylander, 1848 & 23.vi.2017 & $\mathrm{F}$ & 3968 & http:/ / osac.oregonstate.edu/SP/OSAC_0001226842 \\
\hline Bombus melanopygus Nylander, 1848 & 23.vi.2017 & $\mathrm{F}$ & 4076 & http:/ / osac.oregonstate.edu/SP/OSAC_0001226843 \\
\hline Bombus mixtus Cresson, 1878 & 18.v.2016 & $\mathrm{F}$ & 107 & http:/ / osac.oregonstate.edu/SP/OSAC_0001226846 \\
\hline Bombus mixtus Cresson, 1878 & 25.v.2016 & $\mathrm{F}$ & 141 & http:/ / osac.oregonstate.edu/SP/OSAC_0001226848 \\
\hline Bombus mixtus Cresson, 1878 & 25.v.2016 & $\mathrm{F}$ & 147 & http:/ / osac.oregonstate.edu/SP/OSAC_0001226849 \\
\hline Bombus mixtus Cresson, 1878 & 07.vii.2016 & $\mathrm{M}$ & 450 & http:/ / osac.oregonstate.edu/SP/OSAC_0001226854 \\
\hline Bombus mixtus Cresson, 1878 & 11.viii.2016 & $\mathrm{F}$ & 1111 & http:/ / osac.oregonstate.edu/SP/OSAC_0001226847 \\
\hline Bombus mixtus Cresson, 1878 & 18.v.2017 & $\mathrm{F}$ & 3694 & http:// osac.oregonstate.edu/SP/OSAC_0001226850 \\
\hline Bombus mixtus Cresson, 1878 & 23.vi.2017 & M & 4075 & http:/ / osac.oregonstate.edu/SP/OSAC_0001226851 \\
\hline Bombus mixtus Cresson, 1878 & 22.vi.2017 & M & 4256 & http:/ / osac.oregonstate.edu/SP/OSAC_0001226852 \\
\hline Bombus mixtus Cresson, 1878 & 22.vi.2017 & $\mathrm{M}$ & 4310 & http:/ / osac.oregonstate.edu/SP/OSAC_0001226853 \\
\hline Bombus sitkensis Nylander, 1848 & 22.vi.2017 & $\mathrm{F}$ & 4250 & http:/ / osac.oregonstate.edu/SP/OSAC_0001226855 \\
\hline Bombus sitkensis Nylander, 1848 & 22.vi.2017 & $\mathrm{F}$ & 4376 & http:/ / osac.oregonstate.edu/SP/OSAC_0001226856 \\
\hline Bombus vandykei (Frison, 1927) & 23.vi.2017 & $\mathrm{F}$ & 3945 & http:/ / osac.oregonstate.edu/SP/OSAC_0001226857 \\
\hline Bombus vandykei (Frison, 1927) & 23.vi.2017 & M & 3946 & http:/ / osac.oregonstate.edu/SP/OSAC_0001226861 \\
\hline Bombus vandykei (Frison, 1927) & 23.vi.2017 & M & 3947 & http:/ / osac.oregonstate.edu/SP/OSAC_0001226862 \\
\hline Bombus vandykei (Frison, 1927) & 29.vi.2017 & $\mathrm{F}$ & 4466 & http:/ / osac.oregonstate.edu/SP/OSAC_0001226858 \\
\hline Bombus vandykei (Frison, 1927) & 29.vi.2017 & $\mathrm{M}$ & 4513 & http:/ / osac.oregonstate.edu/SP/OSAC_0001226863 \\
\hline Bombus vandykei (Frison, 1927) & 27.vii.2017 & $\mathrm{F}$ & 5323 & http:/ / osac.oregonstate.edu/SP/OSAC_0001226859 \\
\hline Bombus vandykei (Frison, 1927) & 27.vii.2017 & M & 5325 & http:/ / osac.oregonstate.edu/SP/OSAC_0001226864 \\
\hline Bombus vandykei (Frison, 1927) & 27.vii.2017 & $\mathrm{M}$ & 5326 & http:/ / osac.oregonstate.edu/SP/OSAC_0001226865 \\
\hline Bombus vandykei (Frison, 1927) & 27.vii.2017 & $\mathrm{F}$ & 5594 & http:/ / osac.oregonstate.edu/SP/OSAC_0001226860 \\
\hline
\end{tabular}




\begin{tabular}{|c|c|c|c|c|}
\hline Bombus vosnesenskii Radoszkowski, 1862 & 11.viii.2016 & $\mathrm{F}$ & 1110 & http:/ / osac.oregonstate.edu/SP/OSAC_0001226866 \\
\hline Bombus vosnesenskii Radoszkowski, 1862 & 11.viii.2016 & $\mathrm{F}$ & 1112 & http:/ / osac.oregonstate.edu/SP/OSAC_0001226867 \\
\hline Bombus vosnesenskii Radoszkowski, 1862 & 11.viii.2016 & $\mathrm{F}$ & 1113 & http:/ / osac.oregonstate.edu/SP/OSAC_0001226868 \\
\hline Bombus vosnesenskii Radoszkowski, 1862 & 11.viii.2016 & $\mathrm{M}$ & 1114 & http:/ / osac.oregonstate.edu/SP/OSAC_0001226871 \\
\hline Bombus vosnesenskii Radoszkowski, 1862 & 11.viii.2016 & M & 1115 & http:/ / osac.oregonstate.edu/SP/OSAC_0001226872 \\
\hline Bombus vosnesenskii Radoszkowski, 1862 & 11.viii.2016 & $\mathrm{F}$ & 1116 & http:/ / osac.oregonstate.edu/SP/OSAC_0001226869 \\
\hline Bombus vosnesenskii Radoszkowski, 1862 & 11.viii.2016 & M & 1117 & http:/ / osac.oregonstate.edu/SP/OSAC_0001226873 \\
\hline Bombus vosnesenskii Radoszkowski, 1862 & 11.viii.2016 & M & 1118 & http:/ / osac.oregonstate.edu/SP/OSAC_0001226874 \\
\hline Bombus vosnesenskii Radoszkowski, 1862 & 11.viii.2016 & $\mathrm{F}$ & 1119 & http:/ / osac.oregonstate.edu/SP/OSAC_0001226870 \\
\hline Bombus vosnesenskii Radoszkowski, 1862 & 11.viii.2016 & M & 1120 & http:/ / osac.oregonstate.edu/SP/OSAC_0001226875 \\
\hline Ceratina tejonensis Cresson, 1864 & 30.vi.2016 & $\mathrm{F}$ & 466 & http:/ / osac.oregonstate.edu/SP/OSAC_0001226876 \\
\hline Ceratina tejonensis Cresson, 1864 & 30.vi.2016 & $\mathrm{F}$ & 467 & http:/ / osac.oregonstate.edu/SP /OSAC_0001226877 \\
\hline Ceratina tejonensis Cresson, 1864 & 30.vi.2016 & $\mathrm{F}$ & 468 & http:/ / osac.oregonstate.edu/SP/OSAC_0001226878 \\
\hline Ceratina tejonensis Cresson, 1864 & 23.vi.2017 & M & 4086 & http:/ / osac.oregonstate.edu/SP/OSAC_0001226879 \\
\hline Eucera morphospecies \#1 & 19.v.2016 & $\mathrm{F}$ & 44 & http:/ / osac.oregonstate.edu/SP/OSAC_0001226906 \\
\hline Eucera morphospecies \#2 & 18.v.2016 & $\mathrm{F}$ & 273 & http:/ / osac.oregonstate.edu/SP/OSAC_0001226907 \\
\hline Eucera cordleyi (Viereck, 1905) & 19.v.2016 & $\mathrm{F}$ & 43 & http:/ / osac.oregonstate.edu/SP/OSAC_0001226898 \\
\hline Eucera cordleyi (Viereck, 1905) & 19.v.2016 & $\mathrm{F}$ & 130 & http:/ / osac.oregonstate.edu/SP/OSAC_0001226897 \\
\hline Eucera cordleyi (Viereck, 1905) & 18.v.2016 & M & 179 & http:/ / osac.oregonstate.edu/SP/OSAC_0001226902 \\
\hline Eucera cordleyi (Viereck, 1905) & 17.v.2017 & M & 3648 & http:/ / osac.oregonstate.edu/SP/OSAC_0001226903 \\
\hline Eucera cordleyi (Viereck, 1905) & 24.v.2017 & M & 3700 & http:/ / osac.oregonstate.edu/SP/OSAC_0001226904 \\
\hline Eucera cordleyi (Viereck, 1905) & 17.v.2017 & M & 3786 & http:/ / osac.oregonstate.edu/SP/OSAC_0001226905 \\
\hline Eucera cordleyi (Viereck, 1905) & 29.vi.2017 & $\mathrm{F}$ & 4436 & http:/ / osac.oregonstate.edu/SP/OSAC_0001226899 \\
\hline Eucera cordleyi (Viereck, 1905) & 28.vi.2017 & $\mathrm{F}$ & 4506 & http:/ / osac.oregonstate.edu/SP/OSAC_0001226900 \\
\hline Eucera cordleyi (Viereck, 1905) & 28.vi.2017 & $\mathrm{F}$ & 4507 & http:/ / osac.oregonstate.edu/SP/OSAC_0001226901 \\
\hline Melecta pacifica Cresson, 1878 & 19.v.2016 & $\mathrm{F}$ & 45 & http:/ / osac.oregonstate.edu/SP/OSAC_0001227202 \\
\hline Melecta pacifica Cresson, 1878 & 24.v.2017 & $\mathrm{F}$ & 3702 & http:/ / osac.oregonstate.edu/SP/OSAC_0001227201 \\
\hline Melissodes communis Cresson, 1878 & 04.viii.2016 & $\mathrm{F}$ & 1137 & http:/ / osac.oregonstate.edu/SP/OSAC_0001227203 \\
\hline Melissodes communis Cresson, 1878 & 04.viii.2016 & $\mathrm{F}$ & 1840 & http:/ / osac.oregonstate.edu/SP/OSAC_0001227204 \\
\hline Melissodes communis Cresson, 1878 & 04.viii.2016 & $\mathrm{F}$ & 2132 & http:/ / osac.oregonstate.edu/SP/OSAC_0001227205 \\
\hline Melissodes communis Cresson, 1878 & 04.viii.2016 & $\mathrm{F}$ & 2133 & http:/ / osac.oregonstate.edu/SP/OSAC_0001227206 \\
\hline Melissodes communis Cresson, 1878 & 04.viii.2016 & $\mathrm{F}$ & 2134 & http:/ / osac.oregonstate.edu/SP/OSAC_0001227207 \\
\hline Melissodes communis Cresson, 1878 & 02.viii.2017 & M & 4901 & http:/ / osac.oregonstate.edu/SP/OSAC_0001227208 \\
\hline Melissodes communis Cresson, 1878 & 03.viii.2017 & M & 4962 & http:/ / osac.oregonstate.edu/SP/OSAC_0001227209 \\
\hline Melissodes communis Cresson, 1878 & 03.viii.2017 & $\mathrm{M}$ & 4966 & http:/ / osac.oregonstate.edu/SP/OSAC_0001227210 \\
\hline Melissodes communis Cresson, 1878 & 03.viii.2017 & M & 4984 & http:/ / osac.oregonstate.edu/SP /OSAC_0001227211 \\
\hline Melissodes communis Cresson, 1878 & 03.viii.2017 & $\mathrm{M}$ & 4990 & http:/ / osac.oregonstate.edu/SP /OSAC_0001227212 \\
\hline Melissodes lupina Cresson, 1878 & 04.viii.2016 & $\mathrm{F}$ & 2140 & http:/ / osac.oregonstate.edu/SP/OSAC_0001227213 \\
\hline Melissodes metenua Cockerell, 1924 & 29.vi.2017 & $\mathrm{F}$ & 4468 & http:/ / osac.oregonstate.edu/SP/OSAC_0001227214 \\
\hline Melissodes metenua Cockerell, 1924 & 02.viii.2017 & $\mathrm{F}$ & 4900 & http:/ / osac.oregonstate.edu/SP/OSAC_0001227215 \\
\hline Melissodes metenua Cockerell, 1924 & 03.viii.2017 & $\mathrm{F}$ & 4938 & http:/ / osac.oregonstate.edu/SP/OSAC_0001227216 \\
\hline Melissodes metenua Cockerell, 1924 & 03.viii.2017 & $\mathrm{F}$ & 4939 & http:/ / osac.oregonstate.edu/SP/OSAC_0001227217 \\
\hline
\end{tabular}




\begin{tabular}{|c|c|c|c|c|}
\hline Melissodes metenua Cockerell, 1924 & 03.viii.2017 & $\mathrm{F}$ & 4999 & http:/ / osac.oregonstate.edu/SP/OSAC_0001227218 \\
\hline Melissodes microsticta Cockerell, 1905 & 04.viii.2016 & $\mathrm{F}$ & 1841 & http:/ / osac.oregonstate.edu/SP/OSAC_0001227219 \\
\hline Melissodes microsticta Cockerell, 1905 & 03.viii.2016 & $\mathrm{F}$ & 1858 & http:/ / osac.oregonstate.edu/SP/OSAC_0001227220 \\
\hline Melissodes microsticta Cockerell, 1905 & 04.viii.2016 & $\mathrm{F}$ & 2138 & http:/ / osac.oregonstate.edu/SP/OSAC_0001227221 \\
\hline Melissodes microsticta Cockerell, 1905 & 04.viii.2016 & $\mathrm{F}$ & 2193 & http:/ / osac.oregonstate.edu/SP/OSAC_0001227222 \\
\hline Melissodes microsticta Cockerell, 1905 & 03.viii.2016 & $\mathrm{F}$ & 2364 & http:/ / osac.oregonstate.edu/SP/OSAC_0001227223 \\
\hline Melissodes microsticta Cockerell, 1905 & 27.vii.2017 & $\mathrm{M}$ & 5392 & http:/ / osac.oregonstate.edu/SP/OSAC_0001227224 \\
\hline Melissodes microsticta Cockerell, 1905 & 26.vii.2017 & $\mathrm{M}$ & 5456 & http:/ / osac.oregonstate.edu/SP/OSAC_0001227225 \\
\hline Melissodes microsticta Cockerell, 1905 & 03.viii.2017 & $\mathrm{M}$ & 5689 & http:/ / osac.oregonstate.edu/SP/OSAC_0001227226 \\
\hline Melissodes rivalis Cresson, 1872 & 08.vii.2016 & $\mathrm{M}$ & 383 & http:/ / osac.oregonstate.edu/SP/OSAC_0001227236 \\
\hline Melissodes rivalis Cresson, 1872 & 04.viii.2016 & $\mathrm{F}$ & 1141 & http:/ / osac.oregonstate.edu/SP/OSAC_0001227227 \\
\hline Melissodes rivalis Cresson, 1872 & 04.viii.2016 & $\mathrm{F}$ & 1587 & http:/ / osac.oregonstate.edu/SP/OSAC_0001227228 \\
\hline Melissodes rivalis Cresson, 1872 & 04.viii.2016 & $\mathrm{F}$ & 2139 & http:/ / osac.oregonstate.edu/SP/OSAC_0001227229 \\
\hline Melissodes rivalis Cresson, 1872 & 11.viii.2016 & $\mathrm{F}$ & 2926 & http:/ / osac.oregonstate.edu/SP/OSAC_0001227230 \\
\hline Melissodes rivalis Cresson, 1872 & 03.viii.2016 & $\mathrm{M}$ & 2950 & http:/ / osac.oregonstate.edu/SP/OSAC_0001227232 \\
\hline Melissodes rivalis Cresson, 1872 & 08.ix.2016 & $\mathrm{M}$ & 3621 & http:/ / osac.oregonstate.edu/SP/OSAC_0001227233 \\
\hline Melissodes rivalis Cresson, 1872 & 08.ix.2016 & $\mathrm{M}$ & 3622 & http:/ / osac.oregonstate.edu/SP/OSAC_0001227234 \\
\hline Melissodes rivalis Cresson, 1872 & 08.ix.2016 & $\mathrm{M}$ & 3623 & http:/ / osac.oregonstate.edu/SP/OSAC_0001227235 \\
\hline Melissodes rivalis Cresson, 1872 & 02.viii.2017 & $\mathrm{F}$ & 4854 & http:/ / osac.oregonstate.edu/SP/OSAC_0001227231 \\
\hline Melissodes morphospecies \#1 & 02.viii.2017 & $\mathrm{M}$ & 4897 & http:/ / osac.oregonstate.edu/SP/OSAC_0001227237 \\
\hline Melissodes morphospecies \#1 & 02.viii.2017 & M & 4898 & http:/ / osac.oregonstate.edu/SP/OSAC_0001227238 \\
\hline Melissodes morphospecies \#1 & 03.viii.2017 & M & 4963 & http:/ / osac.oregonstate.edu/SP/OSAC_0001227239 \\
\hline Melissodes morphospecies \#1 & 02.viii.2017 & $\mathrm{M}$ & 5213 & http:/ / osac.oregonstate.edu/SP/OSAC_0001227240 \\
\hline Melissodes morphospecies \#1 & 27.vii.2017 & $\mathrm{M}$ & 5328 & http:/ / osac.oregonstate.edu/SP/OSAC_0001227241 \\
\hline Xylocopa tabaniformis Smith, 1854 & 19.v.2016 & $\mathrm{F}$ & 10 & http:/ / osac.oregonstate.edu/SP/OSAC_0001227281 \\
\hline Xylocopa tabaniformis Smith, 1854 & 19.v.2016 & $\mathrm{F}$ & 11 & http:/ / osac.oregonstate.edu/SP/OSAC_0001227282 \\
\hline Xylocopa tabaniformis Smith, 1854 & 19.v.2016 & $\mathrm{M}$ & 109 & http:/ / osac.oregonstate.edu/SP/OSAC_0001227286 \\
\hline Xylocopa tabaniformis Smith, 1854 & 19.v.2016 & $\mathrm{M}$ & 110 & http:/ / osac.oregonstate.edu/SP/OSAC_0001227287 \\
\hline Xylocopa tabaniformis Smith, 1854 & 19.v.2016 & $\mathrm{M}$ & 111 & http:/ / osac.oregonstate.edu/SP/OSAC_0001227288 \\
\hline Xylocopa tabaniformis Smith, 1854 & 19.v.2016 & $\mathrm{M}$ & 112 & http:/ / osac.oregonstate.edu/SP/OSAC_0001227289 \\
\hline Xylocopa tabaniformis Smith, 1854 & 19.v.2016 & $\mathrm{M}$ & 113 & http:/ / osac.oregonstate.edu/SP/OSAC_0001227290 \\
\hline Xylocopa tabaniformis Smith, 1854 & 11.viii.2016 & $\mathrm{F}$ & 1102 & http:/ / osac.oregonstate.edu/SP/OSAC_0001227283 \\
\hline Xylocopa tabaniformis Smith, 1854 & 11.viii.2016 & $\mathrm{F}$ & 1103 & http:/ / osac.oregonstate.edu/SP/OSAC_0001227284 \\
\hline Xylocopa tabaniformis Smith, 1854 & 11.viii.2016 & $\mathrm{F}$ & 1104 & http:/ / osac.oregonstate.edu/SP/OSAC_0001227285 \\
\hline \multicolumn{5}{|c|}{ COLLETIDAE } \\
\hline Colletes fulgidus Swenk, 1904 & 11.viii.2016 & $\mathrm{F}$ & 954 & http:/ / osac.oregonstate.edu/SP/OSAC_0001226882 \\
\hline Colletes fulgidus Swenk, 1904 & 11.viii.2016 & $\mathrm{F}$ & 955 & http:/ / osac.oregonstate.edu/SP/OSAC_0001226883 \\
\hline Colletes fulgidus Swenk, 1904 & 11.viii.2016 & $\mathrm{F}$ & 1123 & http:/ / osac.oregonstate.edu/SP/OSAC_0001226880 \\
\hline Colletes fulgidus Swenk, 1904 & 11.viii.2016 & $\mathrm{M}$ & 2886 & http:/ / osac.oregonstate.edu/SP/OSAC_0001226884 \\
\hline Colletes fulgidus Swenk, 1904 & 10.viii.2016 & $\mathrm{F}$ & 2983 & http:/ / osac.oregonstate.edu/SP/OSAC_0001226881 \\
\hline Colletes kincaidii Cockerell, 1898 & 04.viii.2016 & M & 1924 & http:/ / osac.oregonstate.edu/SP/OSAC_0001226885 \\
\hline Colletes kincaidii Cockerell, 1898 & 03.viii.2016 & $\mathrm{M}$ & 2365 & http:/ / osac.oregonstate.edu/SP/OSAC_0001226886 \\
\hline
\end{tabular}




\begin{tabular}{|c|c|c|c|c|}
\hline Colletes kincaidii Cockerell, 1898 & 03.viii.2017 & M & 4844 & http:/ / osac.oregonstate.edu/SP/OSAC_0001226887 \\
\hline Hylaeus affinis (Smith, 1853) & 07.vii.2016 & $\mathrm{F}$ & 616 & http:/ / osac.oregonstate.edu/SP/OSAC_0001226942 \\
\hline Hylaeus affinis (Smith, 1853) & 07.vii.2016 & $\mathrm{F}$ & 634 & http:/ / osac.oregonstate.edu/SP/OSAC_0001226943 \\
\hline Hylaeus affinis (Smith, 1853) & 11.viii.2016 & $\mathrm{F}$ & 957 & http:/ / osac.oregonstate.edu/SP/OSAC_0001226944 \\
\hline Hylaeus affinis (Smith, 1853) & 10.viii.2016 & $\mathrm{F}$ & 2998 & http:/ / osac.oregonstate.edu/SP/OSAC_0001226940 \\
\hline Hylaeus affinis (Smith, 1853) & 08.ix.2016 & $\mathrm{F}$ & 3400 & http:/ / osac.oregonstate.edu/SP/OSAC_0001226941 \\
\hline Hylaeus episcopalis (Cockerell, 1896) & 11.viii.2016 & $\mathrm{F}$ & 959 & http:/ / osac.oregonstate.edu/SP/OSAC_0001226945 \\
\hline Hylaeus nevadensis (Cockerell, 1896) & 11.viii.2016 & $\mathrm{F}$ & 986 & http:/ / osac.oregonstate.edu/SP/OSAC_0001226948 \\
\hline Hylaeus nevadensis (Cockerell, 1896) & 10.viii.2016 & $\mathrm{F}$ & 2991 & http:/ / osac.oregonstate.edu/SP/OSAC_0001226946 \\
\hline Hylaeus nevadensis (Cockerell, 1896) & 27.vii.2017 & $\mathrm{F}$ & 5346 & http:/ / osac.oregonstate.edu/SP/OSAC_0001226947 \\
\hline Hylaeus rugulosus (Perkins, 1899) & 03.viii.2016 & $\mathrm{F}$ & 2367 & http:/ / osac.oregonstate.edu/SP/OSAC_0001226949 \\
\hline Hylaeus rugulosus (Perkins, 1899) & 11.viii.2016 & F & 2891 & http:/ / osac.oregonstate.edu/SP/OSAC_0001226950 \\
\hline Hylaeus timberlakei Snelling, 1970 & 04.viii.2016 & $\mathrm{M}$ & 1554 & http:/ / osac.oregonstate.edu/SP/OSAC_0001226951 \\
\hline Hylaeus verticalis (Cresson, 1869) & 29.vi.2017 & $\bar{F}$ & 4571 & http:/ / osac.oregonstate.edu/SP/OSAC_0001226952 \\
\hline Hylaeus wootoni (Cockerell, 1896) & 08.vii.2016 & $\mathrm{M}$ & 358 & http:/ / osac.oregonstate.edu/SP/OSAC_0001226954 \\
\hline Hylaeus wootoni (Cockerell, 1896) & 28.vi.2017 & $\mathrm{F}$ & 4721 & http:/ / osac.oregonstate.edu/SP/OSAC_0001226953 \\
\hline Hylaeus wootoni (Cockerell, 1896) & 03.viii.2017 & $\mathrm{M}$ & 4941 & http:/ / osac.oregonstate.edu/SP/OSAC_0001226955 \\
\hline Hylaeus wootoni (Cockerell, 1896) & 27.vii.2017 & $\mathrm{M}$ & 5621 & http:/ / osac.oregonstate.edu/SP/OSAC_0001226956 \\
\hline Hylaeus morphospecies \#1 & 11.viii.2016 & $\mathrm{F}$ & 963 & http:/ / osac.oregonstate.edu/SP/OSAC_0001226937 \\
\hline Hylaeus morphospecies \#2 & 08.vii.2016 & M & 354 & http:/ / osac.oregonstate.edu/SP/OSAC_0001226938 \\
\hline Hylaeus morphospecies \#2 & 29.vi.2017 & $\mathrm{M}$ & 4759 & http:/ / osac.oregonstate.edu/SP/OSAC_0001226939 \\
\hline \multicolumn{5}{|c|}{ HALICTIDAE } \\
\hline Dufourea calochorti (Cockerell, 1924) & 30.vi.2016 & $\mathrm{F}$ & 540 & http:/ / osac.oregonstate.edu/SP/OSAC_0001226896 \\
\hline Halictus confusus Smith, 1853 & 29.vi.2017 & $\mathrm{F}$ & 4652 & http:/ / osac.oregonstate.edu/SP/OSAC_0001226908 \\
\hline Halictus farinosus Smith, 1853 & 26.v.2016 & $\mathrm{F}$ & 169 & http:/ / osac.oregonstate.edu/SP/OSAC_0001226909 \\
\hline Halictus farinosus Smith, 1853 & 26.v.2016 & $\mathrm{F}$ & 170 & http:/ / osac.oregonstate.edu/SP /OSAC_0001226910 \\
\hline Halictus farinosus Smith, 1853 & 26.v.2016 & $\mathrm{F}$ & 171 & http:/ / osac.oregonstate.edu/SP/OSAC_0001226911 \\
\hline Halictus farinosus Smith, 1853 & 04.viii.2016 & $\mathrm{M}$ & 1138 & http:/ / osac.oregonstate.edu/SP/OSAC_0001226914 \\
\hline Halictus farinosus Smith, 1853 & 03.viii.2016 & $\mathrm{F}$ & 1859 & http:/ / osac.oregonstate.edu/SP/OSAC_0001226912 \\
\hline Halictus farinosus Smith, 1853 & 04.viii.2016 & $\mathrm{F}$ & 1922 & http:/ / osac.oregonstate.edu/SP/OSAC_0001226913 \\
\hline Halictus farinosus Smith, 1853 & 04.viii.2016 & $\mathrm{M}$ & 1923 & http:/ / osac.oregonstate.edu/SP/OSAC_0001226915 \\
\hline Halictus farinosus Smith, 1853 & 04.viii.2016 & M & 2297 & http:/ / osac.oregonstate.edu/SP/OSAC_0001226916 \\
\hline Halictus farinosus Smith, 1853 & 01.ix.2016 & M & 3515 & http:/ / osac.oregonstate.edu/SP/OSAC_0001226917 \\
\hline Halictus farinosus Smith, 1853 & 01.ix.2017 & M & 6107 & http:/ / osac.oregonstate.edu/SP/OSAC_0001226918 \\
\hline Halictus ligatus Say, 1837 & 01.ix.2017 & $\mathrm{F}$ & 6108 & http:/ / osac.oregonstate.edu/SP/OSAC_0001226919 \\
\hline Halictus ligatus Say, 1837 & 01.ix.2017 & $\mathrm{F}$ & 6359 & http:/ / osac.oregonstate.edu/SP/OSAC_0001226920 \\
\hline Halictus rubicundus (Christ, 1791) & 07.vii.2016 & F & 633 & http:/ / osac.oregonstate.edu/SP/OSAC_0001226924 \\
\hline Halictus rubicundus (Christ, 1791) & 08.ix.2016 & $\mathrm{F}$ & 3397 & http:/ / osac.oregonstate.edu/SP/OSAC_0001226921 \\
\hline Halictus rubicundus (Christ, 1791) & 22.vi.2017 & $\mathrm{F}$ & 4377 & http:/ / osac.oregonstate.edu/SP/OSAC_0001226922 \\
\hline Halictus rubicundus (Christ, 1791) & 02.viii.2017 & $\mathrm{F}$ & 5071 & http:/ / osac.oregonstate.edu/SP/OSAC_0001226923 \\
\hline Halictus tripartitus Cockerell, 1895 & 11.viii.2016 & $\mathrm{F}$ & 1124 & http:/ / osac.oregonstate.edu/SP/OSAC_0001226925 \\
\hline Halictus tripartitus Cockerell, 1895 & 11.viii.2016 & $\mathrm{F}$ & 1125 & http:/ / osac.oregonstate.edu/SP/OSAC_0001226926 \\
\hline
\end{tabular}




\begin{tabular}{|c|c|c|c|c|}
\hline Halictus tripartitus Cockerell, 1895 & 1.viii.2016 & $\mathrm{F}$ & 1126 & http:/ / osac.oregonstate.edu/SP/OSAC_0001226927 \\
\hline Halictus tripartitus Cockerell, 1895 & iii.2016 & $\mathrm{F}$ & 130 & http:/ / osac.oregonstate.edu/SP/OSAC_0001226928 \\
\hline Halictus tripartitus Cockerell, 1895 & 16 & $\mathrm{~F}$ & 31 & http:/ / osac.oregonstate.edu/SP/OSAC_0001226929 \\
\hline Lasioglossum (Dialictus) morphospecies \#1 & vi.2016 & M & 648 & http:/ / osac.oregonstate.edu/SP/OSAC_0001226963 \\
\hline asioglossum (Dialictus) morphospecies \#1 & 16 & $\mathrm{~F}$ & 27 & (1) \\
\hline Lasioglossum (Dialictus) morp & & $\mathrm{F}$ & 1555 & http:/ / osac.oregonstate.ed \\
\hline Lasioglossum (Dialictus) morphospecies \#1 & 2016 & $\mathrm{~F}$ & 1556 & http:/ / osac.oregonstate.edu/SP/OSAC_0001226960 \\
\hline Lasioglossum (Dialictus) morphospecies \#1 & 16 & $\mathrm{~F}$ & 1557 & http:/ / osac.oregonstate.edu/SP/OSAC_0001226961 \\
\hline Lasioglossum (Dialictus) morphospecies \#1 & & $\mathrm{M}$ & 3154 & http:/ / osac.oregonstate.e \\
\hline Lasioglossum (Dialictus) mor & 17 & $\mathrm{~F}$ & 5225 & http:/ / osac.oregonstate.edu/SP/OSAC_0001226957 \\
\hline Lasiog & & $\mathrm{F}$ & 150 & http:/ / osac.oregonstate.edu/SP/OSAC_0001226980 \\
\hline Lasio & & $\mathrm{F}$ & 151 & http:/ / osac.oregonstate.edu/SP/OSAC_0001226981 \\
\hline Lasic & & $\mathrm{F}$ & 153 & http:/ / osac.oregonstate.edu/SP/OSAC_0001226982 \\
\hline Lasioglossum (Dialictus) $\mathrm{n}$ & & $\mathrm{F}$ & 154 & http:/ / osac.oregonstate.edu/SP/OSA \\
\hline Lasioglossum (Dialictus) mo & & $\mathrm{F}$ & 155 & http:/ / osac.oregonstate.edu/SP/OSAC_0001226984 \\
\hline Lasioglossum (Dialictus) mc & 3.viii.2016 & $\mathrm{M}$ & 1678 & http:// osac.oregonstate.edu/SP/OSAC_0001226985 \\
\hline Lasioglossum (Dialictus) morphospecies \#3 & viii.2016 & $\mathrm{F}$ & 8 & http:/ / osac.oregonstate.e \\
\hline Lasioglossum (Dialictus) morphospecies \#3 & t.viii.2016 & $\mathrm{F}$ & 2301 & 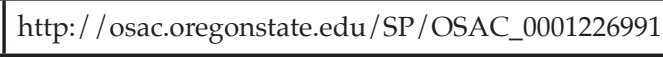 \\
\hline Lasioglossum (Dialictus) morphospecies \#3 & 3.viii.2016 & $\mathrm{F}$ & 2366 & http:/ / osac.o \\
\hline Lasioglossum (Dialictus) morphospecies \#3 & viii.2016 & $\mathrm{F}$ & 2985 & http:/ / osac.oregonstate.edu/SP/OSAC \\
\hline Lasioglossum (Dialictus) morphospecies \#5 & 16 & $\mathrm{~F}$ & 158 & http:/ / osac.oregonstate.edu/SP/OSAC \\
\hline Lasioglossum (Dialictus) morp & i.2016 & $\mathrm{F}$ & 1625 & http:/ / osac.oregonstate.edu/SP/OSAC_0001226996 \\
\hline Lasioglossum (Dialictus) morphospecies \#5 & viii.2016 & $\mathrm{F}$ & 1687 & http:/ / osac.oregonstate.edu/SP/OSAC_0001226997 \\
\hline Lasioglossum (Dialictus) morp & viii.2016 & $\mathrm{F}$ & 1719 & http:/ / osac.oregonstate.edu/SP/OSAC_0001226998 \\
\hline Lasioglossum (Dialictus) mor & i. 2016 & $\mathrm{~F}$ & 1845 & http:/ / osac.oregonstate.edu/SP/OSAC_0001226999 \\
\hline Lasioglossum (Dialictus) morphospecies \#6 & & $\mathrm{F}$ & 1604 & http:/ / osac.oregonstate.edu/SP/OSAC_0001227000 \\
\hline Lasioglossum (Dialictus) morphospecies \#6 & viii.2016 & $\mathrm{F}$ & 1628 & tp: / / osac.oregonstate.edu/SP/OSA \\
\hline Lasioglossum (Dialictus) morphospecies \#6 & viii.2016 & $\mathrm{F}$ & 9 & ttp: / / osac.oregonstate.edu/SP/OS \\
\hline Lasioglossum (Dialictus) mor & & $\mathrm{F}$ & 1667 & http:/ / osac.oregonst \\
\hline Lasioglossum (Dialictus) $\mathrm{n}$ & 16 & $\mathrm{~F}$ & 1681 & http:/ / osac.oregor \\
\hline Lasioglossum (Dialictus) morph & 16 & $\mathrm{~F}$ & 1671 & http:/ / osac.oregonstat \\
\hline Lasioglossum (Dialictus) morphosp & 03.viii.2016 & $\mathrm{F}$ & 1721 & http:/ / osac.oregonstate.edu/SP/OSAC_0001227006 \\
\hline Lasioglossum (Dialictus) morphospecies \#7 & 03.viii.2016 & $\mathrm{F}$ & 1726 & http:/ / osac.oregonstate.edu/SP/OSAC_0001227007 \\
\hline Lasioglossum (Dialictus) morphospecies \#7 & 03.viii.2016 & $\mathrm{F}$ & 1883 & http:/ / osac.oregonstate.edu/SP/OSAC_0001227008 \\
\hline Lasioglossum (Dialictus) morphospecies \#7 & 03.viii.2016 & $\mathrm{F}$ & 2100 & http:/ / osac.oregonstate.edu/SP/OSAC_0001227009 \\
\hline Lasioglossum (Dialictus) morphospecies \#9 & 31.viii.2017 & $\mathrm{F}$ & 6220 & http:/ / osac.oregonstate.edu/SP/OSAC_0001227010 \\
\hline Lasioglossum (Dialictus) morphospecies \#10 & 31.viii.2016 & $\mathrm{F}$ & 3067 & http:/ / osac.oregonstate.edu/SP/OSAC_000122 \\
\hline Lasioglossum (Dialictus) morphospecies \#11 & 08.ix.2016 & $\mathrm{F}$ & 3464 & http:/ / osac.oregonstate.edu/SP/OSAC_0001226965 \\
\hline Lasioglossum (Dialictus) morphospecies \#12 & 07.ix.2016 & $\mathrm{M}$ & 3442 & http:/ / osac.oregonstate.edu/SP/OSAC_0001226966 \\
\hline Lasioglossum (Dialictus) morphospecies \#13 & 01.ix.2017 & $\mathrm{M}$ & 6109 & http:/ / osac.oregonstate.edu/SP/OSAC_0001226967 \\
\hline Lasioglossum (Dialictus) morphospecies \#15 & 04.viii.2016 & $\mathrm{F}$ & 1632 & http:/ / osac.oregonstate.edu/SP/OSAC_0001226968 \\
\hline Lasioglossum (Dialictus) morphospecies \#15 & 03.viii.2016 & $\mathrm{M}$ & 1682 & http:/ / osac.oregonstate.edu/SP/OSAC_0001226970 \\
\hline
\end{tabular}




\begin{tabular}{|c|c|c|c|c|}
\hline Lasioglossum (Dialictus) morphospecies \#15 & 03.viii.2016 & $\mathrm{M}$ & 1702 & http:/ / osac.oregonstate.edu/SP/OSAC_0001226971 \\
\hline Lasioglossum (Dialictus) morphospecies \#15 & 03.viii.2016 & $\mathrm{M}$ & 1710 & http:/ / osac.oregonstate.edu/SP/OSAC_0001226972 \\
\hline Lasioglossum (Dialictus) morphospecies \#15 & 03.viii.2016 & $\mathrm{M}$ & 1722 & http:/ / osac.oregonstate.edu/SP/OSAC_0001226973 \\
\hline Lasioglossum (Dialictus) morphospecies \#15 & 03.viii.2016 & $\mathrm{M}$ & 1727 & http:/ / osac.oregonstate.edu/SP/OSAC_0001226974 \\
\hline Lasioglossum (Dialictus) morphospecies \#15 & 03.viii.2016 & $\mathrm{F}$ & 2078 & http:/ / osac.oregonstate.edu/SP/OSAC_0001226969 \\
\hline Lasioglossum (Dialictus) morphospecies \#17 & 03.viii.2016 & $\mathrm{M}$ & 1716 & http:/ / osac.oregonstate.edu/SP/OSAC_0001226975 \\
\hline Lasioglossum (Dialictus) morphospecies \#17 & 03.viii.2016 & $\mathrm{M}$ & 1730 & http:/ / osac.oregonstate.edu/SP/OSAC_0001226976 \\
\hline Lasioglossum (Dialictus) morphospecies \#17 & 03.viii.2016 & $\mathrm{M}$ & 2112 & http:/ / osac.oregonstate.edu/SP/OSAC_0001226977 \\
\hline Lasioglossum (Dialictus) morphospecies \#17 & 11.viii.2016 & M & 2766 & http:/ / osac.oregonstate.edu/SP/OSAC_0001226978 \\
\hline Lasioglossum (Dialictus) morphospecies \#17 & 31.viii.2016 & $\mathrm{M}$ & 3228 & http:/ / osac.oregonstate.edu/SP/OSAC_0001226979 \\
\hline Lasioglossum (Dialictus) morphospecies \#22 & 10.viii.2016 & $\mathrm{M}$ & 2995 & http:/ / osac.oregonstate.edu/SP/OSAC_0001226986 \\
\hline Lasioglossum (Dialictus) morphospecies \#23 & 10.viii. 2016 & $\mathrm{M}$ & 3016 & http:/ / osac.oregonstate.edu/SP/OSAC_0001226987 \\
\hline Lasioglossum (Dialictus) morphospecies \#24 & 02.ix.2016 & $\mathrm{M}$ & 3089 & http:/ / osac.oregonstate.edu/SP/OSAC_0001226988 \\
\hline Lasioglossum (Dialictus) morphospecies \#25 & 02.ix.2016 & $\mathrm{M}$ & 3132 & http:/ / osac.oregonstate.edu/SP/OSAC_0001226989 \\
\hline Lasioglossum (Dialictus) morphospecies \#26 & 03.viii.2016 & $\mathrm{M}$ & 1709 & http:/ / osac.oregonstate.edu/SP/OSAC_0001226990 \\
\hline Lasioglossum (Evylaeus) morphospecies \#1 & 30.vi.2016 & $\mathrm{F}$ & 536 & http:/ / osac.oregonstate.edu/SP/OSAC_0001227015 \\
\hline Lasioglossum (Evylaeus) morphospecies \#1 & 03.viii.2016 & $\mathrm{F}$ & 1860 & http:/ / osac.oregonstate.edu/SP/OSAC_0001227011 \\
\hline Lasioglossum (Evylaeus) morphospecies \#1 & 03.viii.2016 & $\mathrm{F}$ & 2433 & http:/ / osac.oregonstate.edu/SP/OSAC_0001227012 \\
\hline Lasioglossum (Evylaeus) morphospecies \#1 & 11.viii.2016 & $\mathrm{M}$ & 2910 & http:/ / osac.oregonstate.edu/SP/OSAC_0001227016 \\
\hline Lasioglossum (Evylaeus) morphospecies \#1 & 29.vi.2017 & $\mathrm{F}$ & 4669 & http:/ / osac.oregonstate.edu/SP/OSAC_0001227013 \\
\hline Lasioglossum (Evylaeus) morphospecies \#1 & 27.vii.2017 & $\mathrm{F}$ & 5158 & http:/ / osac.oregonstate.edu/SP/OSAC_0001227014 \\
\hline L. (Hemihalictus) morphospecies \#1 & 25.v.2017 & $\mathrm{F}$ & 3900 & http:/ / osac.oregonstate.edu/SP/OSAC_0001227017 \\
\hline L. (Hemihalictus) morphospecies \#3 & 26.v.2016 & $\mathrm{F}$ & 254 & http:/ / osac.oregonstate.edu/SP/OSAC_0001227018 \\
\hline L. (Lasioglossum) anhypops McGinley, 1986 & 30.vi.2016 & $\mathrm{F}$ & 497 & http:/ / osac.oregonstate.edu/SP/OSAC_0001227020 \\
\hline L. (Lasioglossum) anhypops McGinley, 1986 & 23.vi.2017 & $\mathrm{F}$ & 4211 & http:/ / osac.oregonstate.edu/SP/OSAC_0001227019 \\
\hline L. (Lasioglossum) egregium (Vachal, 1904) & 17.v.2017 & $\mathrm{F}$ & 3655 & http:/ / osac.oregonstate.edu/SP/OSAC_0001227021 \\
\hline L. (Lasioglossum) egregium (Vachal, 1904) & 25.v.2017 & $\mathrm{F}$ & 3898 & http:/ / osac.oregonstate.edu/SP/OSAC_0001227022 \\
\hline L. (Lasioglossum) egregium (Vachal, 1904) & 25.v.2017 & $\mathrm{F}$ & 3910 & http:/ / osac.oregonstate.edu/SP/OSAC_0001227023 \\
\hline L. (Lasioglossum) egregium (Vachal, 1904) & 23.vi.2017 & $\mathrm{F}$ & 4108 & http:/ / osac.oregonstate.edu/SP/OSAC_0001227024 \\
\hline L. (Lasioglossum) egregium (Vachal, 1904) & 29.vi.2017 & $\mathrm{F}$ & 4757 & http:/ / osac.oregonstate.edu/SP/OSAC_0001227025 \\
\hline L. (Lasioglossum) mellipes (Crawford, 1907) & 18.v.2016 & $\mathrm{F}$ & 108 & http:/ / osac.oregonstate.edu/SP/OSAC_0001227026 \\
\hline L. (Lasioglossum) mellipes (Crawford, 1907) & 18.v.2016 & $\mathrm{F}$ & 140 & http:/ / osac.oregonstate.edu/SP/OSAC_0001227027 \\
\hline L. (Lasioglossum) mellipes (Crawford, 1907) & 25.v.2016 & $\mathrm{F}$ & 196 & http:/ / osac.oregonstate.edu/SP/OSAC_0001227028 \\
\hline L. (Lasioglossum) mellipes (Crawford, 1907) & 18.v.2016 & $\mathrm{F}$ & 276 & http:/ / osac.oregonstate.edu/SP/OSAC_0001227029 \\
\hline L. (Lasioglossum) mellipes (Crawford, 1907) & 04.viii.2016 & $\mathrm{M}$ & 1140 & http:/ / osac.oregonstate.edu/SP/OSAC_0001227031 \\
\hline L. (Lasioglossum) mellipes (Crawford, 1907) & 08.ix.2016 & $\mathrm{M}$ & 3456 & http:/ / osac.oregonstate.edu/SP/OSAC_0001227032 \\
\hline L. (Lasioglossum) mellipes (Crawford, 1907) & 08.ix.2016 & $\mathrm{M}$ & 3463 & http:/ / osac.oregonstate.edu/SP/OSAC_0001227033 \\
\hline L. (Lasioglossum) mellipes (Crawford, 1907) & 18.v.2017 & $\mathrm{F}$ & 3696 & http:/ / osac.oregonstate.edu/SP/OSAC_0001227030 \\
\hline L. (Lasioglossum) mellipes (Crawford, 1907) & 27.vii.2017 & $\mathrm{M}$ & 5543 & http:/ / osac.oregonstate.edu/SP/OSAC_0001227034 \\
\hline L. (Lasioglossum) mellipes (Crawford, 1907) & 01.ix.2017 & M & 6094 & http:/ / osac.oregonstate.edu/SP/OSAC_0001227035 \\
\hline L. (Lasioglossum) pacificum (Cockerell, 1898) & 26.v.2016 & $\mathrm{F}$ & 252 & http:/ / osac.oregonstate.edu/SP/OSAC_0001227036 \\
\hline L. (Lasioglossum) pacificum (Cockerell, 1898) & 08.ix.2016 & $\mathrm{M}$ & 3628 & http:/ / osac.oregonstate.edu/SP/OSAC_0001227134 \\
\hline
\end{tabular}




\begin{tabular}{|c|c|c|c|c|}
\hline L. (Lasioglossum) pacificum (Cockerell, 1898) & 17.v.2017 & $\mathrm{F}$ & 3649 & http:/ / osac.oregonstate.edu/SP/OSAC_0001227133 \\
\hline L. (Lasioglossum) sisymbrii (Cockerell, 1895) & 27.v.2016 & $\mathrm{F}$ & 339 & http:/ / osac.oregonstate.edu/SP/OSAC_0001227135 \\
\hline L. (Lasioglossum) sisymbrii (Cockerell, 1895) & 07.ix.2016 & $\mathrm{F}$ & 3452 & http:/ / osac.oregonstate.edu/SP/OSAC_0001227136 \\
\hline L. (Lasioglossum) sisymbrii (Cockerell, 1895) & 23.vi.2017 & $\mathrm{F}$ & 4214 & http:/ / osac.oregonstate.edu/SP/OSAC_0001227137 \\
\hline L. (Lasioglossum) sisymbrii (Cockerell, 1895) & 02.viii.2017 & $\mathrm{F}$ & 5214 & http:/ / osac.oregonstate.edu/SP/OSAC_0001227138 \\
\hline L. (Lasioglossum) titusi (Crawford, 1902) & 04.viii.2016 & $\mathrm{M}$ & 1842 & http:/ / osac.oregonstate.edu/SP/OSAC_0001227139 \\
\hline L. (Lasioglossum) titusi (Crawford, 1902) & 28.vi.2017 & $\mathrm{M}$ & 4508 & http:/ / osac.oregonstate.edu/SP/OSAC_0001227140 \\
\hline L. (Sphecodogastra) morphospecies \#1 & 19.v.2016 & $\mathrm{F}$ & 121 & http:/ / osac.oregonstate.edu/SP/OSAC_0001227141 \\
\hline L. (Sphecodogastra) morphospecies \#1 & 04.viii.2016 & $\mathrm{F}$ & 1598 & http:/ / osac.oregonstate.edu/SP/OSAC_0001227165 \\
\hline L. (Sphecodogastra) morphospecies \#1 & ii.2016 & IV1 & 1843 & http:/ / osac.oregonstate.edu/SP/OSAC_0001227169 \\
\hline L. (Sphecodogastra) morphospeci & ii.2016 & $\mathrm{F}$ & 2668 & http:/ / osac.oregonstate.edu/SP/OSAC_0001227166 \\
\hline L. (Sphecodogastra) morphos & .2016 & $\mathrm{~F}$ & 2972 & http:/ / osac.oregonstate.edu/SP/OSAC_0001227167 \\
\hline L. (Sphecodogastra) morphos & 016 & $\mathrm{~F}$ & 3346 & http:/ / osac.oregonstate.edu/SP/OSAC_0001227168 \\
\hline L. (Sphecodogastra) morphospecies \#1 & 07.ix.2016 & $\mathrm{M}$ & 3465 & / osac.oregonstate.edu/SP/OSAC_0001227170 \\
\hline L. (Sphecodogastra) morphospecies \#1 & 29.vi.2017 & $\mathrm{M}$ & 4635 & / osac.oregonstate.edu/SP/OSAC_0001227171 \\
\hline L. (Sphecodogastra) morphospecies \#1 & 02.viii. 2017 & $\mathrm{M}$ & 5215 & http:/ / osac.oregonstate.edu/SP/OSAC_0001227172 \\
\hline L. (Sphecodogastra) morphospecies \#2 & 11.viii.2016 & $\mathrm{F}$ & 1128 & http:/ / osac.oregonstate.edu/SP/OSAC_0001227173 \\
\hline L. (Sphecodogastra) morphospecies \#2 & 11.viii.2016 & $\mathrm{F}$ & 1129 & http:/ / osac.oregonstate.edu/SP/OSAC_0001227174 \\
\hline L. (Sphecodogastra) morphospecies \#2 & 04.viii.2016 & M & 1151 & http:/ / osac.oregonstate.edu/SP/OSAC_0001227178 \\
\hline L. (Sphecodogastra) morphospecies \#2 & 04.viii.2016 & $\mathrm{F}$ & 1601 & http:/ / osac.oregonstate.edu/SP/OSAC_0001227175 \\
\hline L. (Sphecodogastra) morphospecies \#2 & 04.viii.2016 & $\mathrm{M}$ & 1602 & http:/ / osac.oregonstate.edu/SP/OSAC_0001227179 \\
\hline L. (Sphecodogastra) morphospecies \#2 & 04.viii.2016 & $\mathrm{F}$ & 1605 & http:/ / osac.oregonstate.edu/SP/OSAC_0001227176 \\
\hline L. (Sphecodogastra) morphospecies \#2 & 04.viii.2016 & $\mathrm{F}$ & 1627 & http:/ / osac.oregonstate.edu/SP/OSAC_0001227177 \\
\hline L. (Sphecodogastra) morphospecies \#2 & 04.viii.2016 & $\mathrm{M}$ & 1669 & http:/ / osac.oregonstate.edu/SP/OSAC_0001227180 \\
\hline L. (Sphecodogastra) morphospecies \#2 & 03.viii.2016 & $\mathrm{M}$ & 1881 & http:/ / osac.oregonstate.edu/SP/OSAC_0001227181 \\
\hline L. (Sphecodogastra) morphospecies \#2 & 03.viii.2016 & $\mathrm{M}$ & 1886 & http:/ / osac.oregonstate.edu/SP/OSAC_0001227182 \\
\hline L. (Sphecodogastra) morphospecies \#3 & 25.v.2016 & $\mathrm{F}$ & 224 & http:/ / osac.oregonstate.edu/SP/OSAC_0001227183 \\
\hline L. (Sphecodogastra) morphospecies \#3 & 30.vi.2016 & M & 550 & http:/ / osac.oregonstate.edu/SP/OSAC_0001227184 \\
\hline
\end{tabular}

\section{MEGACHILIDAE}

\begin{tabular}{|c|c|c|c|c|}
\hline Anthidium illustre Cresson, 1879 & 08.vii.2016 & $\mathrm{F}$ & 382 & http:/ / osac.oregonstate.edu/SP/OSAC_0001226792 \\
\hline Anthidium illustre Cresson, 1879 & 23.vi.2017 & $\mathrm{F}$ & 4003 & http:/ / osac.oregonstate.edu/SP/OSAC_0001226793 \\
\hline Anthidium illustre Cresson, 1879 & 23.vi.2017 & $\mathrm{F}$ & 4105 & http:/ / osac.oregonstate.edu/SP/OSAC_0001226794 \\
\hline Anthidium illustre Cresson, 1879 & 29.vi.2017 & $\mathrm{F}$ & 4630 & http:/ / osac.oregonstate.edu/SP/OSAC_0001226795 \\
\hline Anthidium illustre Cresson, 1879 & 02.viii.2017 & $\mathrm{M}$ & 5091 & http:/ / osac.oregonstate.edu/SP/OSAC_0001226796 \\
\hline Anthidium morphospecies \#1 & 11.viii.2016 & $\mathrm{M}$ & 1122 & http:/ / osac.oregonstate.edu/SP/OSAC_0001226797 \\
\hline Anthidium morphospecies \#1 & 29.vi.2017 & M & 4752 & http:/ / osac.oregonstate.edu/SP/OSAC_0001226798 \\
\hline Ashmeadiella cactorum (Cockerell, 1897) & 02.viii.2017 & $\mathrm{F}$ & 5115 & http:/ / osac.oregonstate.edu/SP/OSAC_0001226815 \\
\hline Ashmeadiella cactorum (Cockerell, 1897) & 27.vii.2017 & $\mathrm{M}$ & 5507 & http:/ / osac.oregonstate.edu/SP/OSAC_0001226816 \\
\hline Dianthidium parvum (Cresson, 1878) & 26.vii.2017 & $\mathrm{F}$ & 6059 & http:/ / osac.oregonstate.edu/SP/OSAC_0001226888 \\
\hline Dianthidium pudicum (Cresson, 1879) & 11.viii.2016 & $\mathrm{F}$ & 2909 & http:/ / osac.oregonstate.edu/SP/OSAC_0001226889 \\
\hline Dianthidium pudicum (Cresson, 1879) & 29.vi.2017 & $\mathrm{M}$ & 4602 & http:/ / osac.oregonstate.edu/SP/OSAC_0001226891 \\
\hline Dianthidium pudicum (Cresson, 1879) & 31.viii.2017 & $\mathrm{F}$ & 6165 & http:/ / osac.oregonstate.edu/SP/OSAC_0001226890 \\
\hline
\end{tabular}




\begin{tabular}{|c|c|c|c|c|}
\hline Dianthidium singulare (Cresson, 1879) & 02.viii.2017 & $\mathrm{M}$ & 5211 & http:/ / osac.oregonstate.edu/SP/OSAC_0001226892 \\
\hline Dianthidium ulkei (Cresson, 1878) & 10.viii.2016 & $\mathrm{F}$ & 3012 & http:/ / osac.oregonstate.edu/SP/OSAC_0001226893 \\
\hline Dianthidium ulkei (Cresson, 1878) & 31.viii.2016 & $\mathrm{F}$ & 3223 & http:/ / osac.oregonstate.edu/SP/OSAC_0001226894 \\
\hline Dianthidium ulkei (Cresson, 1878) & 03.viii.2017 & $\mathrm{M}$ & 4940 & http:/ / osac.oregonstate.edu/SP/OSAC_0001226895 \\
\hline Hoplitis albifrons (Kirby, 1837) & 18.v.2016 & $\mathrm{F}$ & 274 & http:/ / osac.oregonstate.edu/SP/OSAC_0001226930 \\
\hline Hoplitis albifrons (Kirby, 1837) & 11.viii.2016 & $\mathrm{F}$ & 2742 & http:/ / osac.oregonstate.edu/SP/OSAC_0001226931 \\
\hline Hoplitis albifrons (Kirby, 1837) & 23.vi.2017 & $\mathrm{F}$ & 3936 & http:/ / osac.oregonstate.edu/SP/OSAC_0001226932 \\
\hline Hoplitis albifrons (Kirby, 1837) & 23.vi.2017 & $\mathrm{F}$ & 3949 & http:/ / osac.oregonstate.edu/SP/OSAC_0001226933 \\
\hline Hoplitis albifrons (Kirby, 1837) & 23.vi.2017 & $\mathrm{F}$ & 3971 & http:/ / osac.oregonstate.edu/SP/OSAC_0001226934 \\
\hline Hoplitis albifrons (Kirby, 1837) & 23.vi.2017 & $\mathrm{M}$ & 4082 & http:/ / osac.oregonstate.edu/SP/OSAC_0001226935 \\
\hline Hoplitis albifrons (Kirby, 1837) & 29.vi.2017 & $\mathrm{M}$ & 4755 & http:/ / osac.oregonstate.edu/SP/OSAC_0001226936 \\
\hline Megachile angelarum Cockerell, 1902 & 01.ix.2017 & $\mathrm{F}$ & 6070 & http:/ / osac.oregonstate.edu/SP/OSAC_0001227185 \\
\hline Megachile angelarum Cockerell, 1902 & 01.ix.2017 & $\mathrm{F}$ & 6358 & http:/ / osac.oregonstate.edu/SP/OSAC_0001227186 \\
\hline Megachile fidelis Cresson, 1878 & 02.viii.2017 & $\mathrm{M}$ & 5093 & http:/ / osac.oregonstate.edu/SP/OSAC_0001227187 \\
\hline Megachile melanophaea Smith, 1853 & 29.vi.2017 & $\mathrm{M}$ & 4649 & http:/ / osac.oregonstate.edu/SP/OSAC_0001227188 \\
\hline Megachile montivaga Cresson, 1878 & 30.vi.2016 & $\mathrm{F}$ & 461 & http:/ / osac.oregonstate.edu/SP/OSAC_0001227192 \\
\hline Megachile montivaga Cresson, 1878 & 04.viii.2016 & $\mathrm{F}$ & 2131 & http:/ / osac.oregonstate.edu/SP/OSAC_0001227189 \\
\hline Megachile montivaga Cresson, 1878 & 11.viii.2016 & $\mathrm{F}$ & 2908 & http:/ / osac.oregonstate.edu/SP/OSAC_0001227190 \\
\hline Megachile montivaga Cresson, 1878 & 07.ix.2016 & $\mathrm{F}$ & 3436 & http:/ / osac.oregonstate.edu/SP/OSAC_0001227191 \\
\hline Megachile montivaga Cresson, 1878 & 03.viii.2017 & $\mathrm{F}$ & 4843 & http:/ / osac.oregonstate.edu/SP/OSAC_0001227193 \\
\hline Megachile perihirta Cockerell, 1898 & 04.viii.2016 & $\mathrm{F}$ & 2130 & http:/ / osac.oregonstate.edu/SP/OSAC_0001227194 \\
\hline Megachile perihirta Cockerell, 1898 & 04.viii.2016 & $\mathrm{F}$ & 2184 & http:/ / osac.oregonstate.edu/SP/OSAC_0001227195 \\
\hline Megachile perihirta Cockerell, 1898 & 31.viii.2016 & $\mathrm{F}$ & 3213 & http:/ / osac.oregonstate.edu/SP/OSAC_0001227196 \\
\hline Megachile perihirta Cockerell, 1898 & 08.ix.2016 & $\mathrm{F}$ & 3393 & http:/ / osac.oregonstate.edu/SP/OSAC_0001227197 \\
\hline Megachile perihirta Cockerell, 1898 & 08.ix.2016 & $\mathrm{F}$ & 3630 & http:/ / osac.oregonstate.edu/SP / OSAC_0001227198 \\
\hline Megachile perihirta Cockerell, 1898 & 03.viii.2017 & $\mathrm{M}$ & 5680 & http:/ / osac.oregonstate.edu/SP/OSAC_0001227199 \\
\hline Megachile pugnata Say, 1837 & 03.viii.2017 & $\mathrm{F}$ & 4961 & http:/ / osac.oregonstate.edu/SP/OSAC_0001227200 \\
\hline Osmia atrocyanea Cockerell, 1897 & 30.vi.2016 & $\mathrm{F}$ & 575 & http:/ / osac.oregonstate.edu/SP / OSAC_0001227247 \\
\hline Osmia atrocyanea Cockerell, 1897 & 17.v.2017 & $\mathrm{F}$ & 3640 & http:/ / osac.oregonstate.edu/SP/OSAC_0001227242 \\
\hline Osmia atrocyanea Cockerell, 1897 & 24.v.2017 & $\mathrm{F}$ & 3716 & http:/ / osac.oregonstate.edu/SP/OSAC_0001227243 \\
\hline Osmia atrocyanea Cockerell, 1897 & 24.v.2017 & $\mathrm{F}$ & 3840 & http:/ / osac.oregonstate.edu/SP /OSAC_0001227244 \\
\hline Osmia atrocyanea Cockerell, 1897 & 23.vi.2017 & $\mathrm{F}$ & 4000 & http:/ / osac.oregonstate.edu/SP /OSAC_0001227245 \\
\hline Osmia atrocyanea Cockerell, 1897 & 23.vi.2017 & $\mathrm{F}$ & 4014 & http:/ / osac.oregonstate.edu/SP /OSAC_0001227246 \\
\hline Osmia bruneri Cockerell, 1897 & 18.v.2017 & $\mathrm{M}$ & 3746 & http:/ / osac.oregonstate.edu/SP /OSAC_0001227248 \\
\hline Osmia cara Cockerell, 1910 & 30.vi.2016 & $\mathrm{F}$ & 574 & http:/ / osac.oregonstate.edu/SP /OSAC_0001227249 \\
\hline Osmia cobaltina Cresson, 1878 & 30.vi.2016 & $\mathrm{F}$ & 493 & http:/ / osac.oregonstate.edu/SP /OSAC_0001227252 \\
\hline Osmia cobaltina Cresson, 1878 & 30.vi.2016 & $\mathrm{F}$ & 576 & http:/ / osac.oregonstate.edu/SP/OSAC_0001227253 \\
\hline Osmia cobaltina Cresson, 1878 & 30.vi.2016 & $\mathrm{F}$ & 646 & http:/ / osac.oregonstate.edu/SP/OSAC_0001227254 \\
\hline Osmia cobaltina Cresson, 1878 & 18.v.2017 & $\mathrm{M}$ & 3744 & http:/ / osac.oregonstate.edu/SP/OSAC_0001227255 \\
\hline Osmia cobaltina Cresson, 1878 & 18.v.2017 & $\mathrm{M}$ & 3745 & http:/ / osac.oregonstate.edu/SP/OSAC_0001227256 \\
\hline Osmia cobaltina Cresson, 1878 & 23.vi.2017 & $\mathrm{F}$ & 3937 & http:/ / osac.oregonstate.edu/SP/OSAC_0001227250 \\
\hline Osmia cobaltina Cresson, 1878 & 23.vi.2017 & $\mathrm{F}$ & 4005 & http:/ / osac.oregonstate.edu/SP/OSAC_0001227251 \\
\hline
\end{tabular}




\begin{tabular}{|c|c|c|c|c|}
\hline Osmia gabrielis Cockerell, 1910 & 27.v.2016 & $\mathrm{F}$ & 302 & http:/ / osac.oregonstate.edu/SP/OSAC_0001227257 \\
\hline Osmia gabrielis Cockerell, 1910 & 29.vi.2016 & $\mathrm{F}$ & 653 & http:/ / osac.oregonstate.edu/SP/OSAC_0001227260 \\
\hline Osmia gabrielis Cockerell, 1910 & 29.vi.2016 & $\mathrm{F}$ & 654 & http:/ / osac.oregonstate.edu/SP/OSAC_0001227261 \\
\hline Osmia gabrielis Cockerell, 1910 & 24.v.2017 & $\mathrm{F}$ & 3701 & http:/ / osac.oregonstate.edu/SP/OSAC_0001227258 \\
\hline Osmia gabrielis Cockerell, 1910 & 22.vi.2017 & $\mathrm{F}$ & 4311 & http:/ / osac.oregonstate.edu/SP/OSAC_0001227259 \\
\hline Osmia montana Cresson, 1864 & 30.vi.2016 & $\mathrm{F}$ & 562 & http:/ / osac.oregonstate.edu/SP/OSAC_0001227262 \\
\hline Osmia visenda Sandhouse 1924 & 23.vi.2017 & $\mathrm{F}$ & 3969 & http:/ / osac.oregonstate.edu/SP/OSAC_0001227276 \\
\hline Osmia morphospecies \#1 & 30.vi.2016 & $\mathrm{F}$ & 543 & http:/ / osac.oregonstate.edu/SP/OSAC_0001227263 \\
\hline Osmia morphospecies \#2 & 29.vi.2017 & $\mathrm{F}$ & 4634 & http:/ / osac.oregonstate.edu/SP/OSAC_0001227267 \\
\hline Osmia morphospecies \#2 & 29.vi.2017 & $\mathrm{F}$ & 4648 & http:/ / osac.oregonstate.edu/SP/OSAC_0001227268 \\
\hline Osmia morphospecies \#2 & 29.vi.2017 & $\mathrm{F}$ & 4664 & http:/ / osac.oregonstate.edu/SP/OSAC_0001227269 \\
\hline Osmia morphospecies \#3 & 30.vi.2016 & $\mathrm{F}$ & 519 & http:// osac.oregonstate.edu/SP/OSAC_0001227270 \\
\hline Osmia morphospecies \#4 & 29.vi.2017 & $\mathrm{F}$ & 4601 & http:// osac.oregonstate.edu/SP/OSAC_0001227271 \\
\hline Osmia morphospecies \#5 & 26.v.2016 & $\mathrm{F}$ & 241 & http:// osac.oregonstate.edu/SP/OSAC_0001227272 \\
\hline Osmia morphospecies \#7 & 18.v.2017 & $\mathrm{M}$ & 3747 & http:/ / osac.oregonstate.edu/SP/OSAC_0001227273 \\
\hline Osmia morphospecies \#8 & 27.v.2016 & $\mathrm{M}$ & 338 & http:// osac.oregonstate.edu/SP/OSAC_0001227274 \\
\hline Osmia morphospecies \#9 & 27.v.2016 & $\mathrm{M}$ & 304 & http:/ / osac.oregonstate.edu/SP/OSAC_0001227275 \\
\hline Osmia morphospecies \#10 & 26.v.2016 & $\mathrm{M}$ & 293 & http:// osac.oregonstate.edu/SP/OSAC_0001227264 \\
\hline Osmia morphospecies \#11 & 19.v.2016 & $\mathrm{M}$ & 84 & http:/ / osac.oregonstate.edu/SP/OSAC_0001227266 \\
\hline Osmia morphospecies \#11 & 26.v.2016 & $\mathrm{M}$ & 253 & http:/ / osac.oregonstate.edu/SP/OSAC_0001227265 \\
\hline
\end{tabular}

\section{Acknowledgments}

Funding provided by the U.S. Bureau of Land Management (Grant \#L16AC00229) as well as the Oregon State University College of Forestry's Mealey/Boise Cascade/Boone and Crockett/Noble Endowment Fund and the Fish and Wildlife Habitat in Managed Forests Research Program.

\section{References}

Galbraith, S.M., Cane, J.H., Moldenke, A.R., Best, L.R., and J.W. Rivers. In revision. Salvage logging in severely burned mixed-conifer forest reduces wild bee diversity. Forest Ecosystems and Management 453, p. 117622.

Galbraith, S.M., J.H. Cane, A.R. Moldenke, and J.W. Rivers. 2019. Wild bee diversity increases with local fire severity in a fire-prone landscape. Ecosphere, 10(4), pp.1-19.

Hurd, P.D., and J.S. Moure. 1963. A classification of the large carpenter bees (Xylocopini, Hymenoptera: Apoidea.) University of California Publications in Entomology 29: 1-365.

Michener, C. D. 2007. The bees of the world. Johns Hopkins University Press, Baltimore, Maryland, USA.

Roberts, R.B., 1973. Bees of Northwestern America: Halictus (Hymenoptera: Halictidae). Technical Bulletin 126, Agricultural Experiment Station, Oregon State University, Corvallis, Oregon.

Stephen, W.P., G.E. Bohart, and P.F. Torchio. 1969. The biology and external morphology of bees; with a synopsis of the genera of Northwestern America. Agricultural Experiment Station, Oregon State University, Oregon, USA.

Williams, P. H., Thorp, R. W., Richardson, L. L., \& Colla, S. R. (2014). Bumble bees of North America: an identification guide. Princeton University Press. 